\title{
Some New Explicit Values of Quotients of Theta-Function $\phi(q)$ and Applications to Ramanujan's Continued Fractions
}

\author{
Nipen Saikia \\ Department of Mathematics, Rajiv Gandhi University, Rono Hills, Doimukh, Arunachal Pradesh 791112, India \\ Correspondence should be addressed to Nipen Saikia; nipennak@yahoo.com
}

Received 14 November 2012; Accepted 23 January 2013

Academic Editor: Nikolai Tarkhanov

Copyright (C) 2013 Nipen Saikia. This is an open access article distributed under the Creative Commons Attribution License, which permits unrestricted use, distribution, and reproduction in any medium, provided the original work is properly cited.

We find some new explicit values of the parameter $h_{k, n}$ for positive real numbers $k$ and $n$ involving Ramanujan's theta-function $\phi(q)$ and give some applications of these new values for the explicit evaluations of Ramanujan's continued fractions. In the process, we also establish two new identities for $\phi(q)$ by using modular equations.

\section{Introduction}

For $q:=e^{2 \pi i z}, \operatorname{Im}(z)>0$, define Ramanujan's theta-function $\phi(q)$ as

$$
\phi(q):=\sum_{n=-\infty}^{\infty} q^{n^{2}}=\vartheta_{3}(0,2 z)
$$

where $\vartheta_{3}[1$, page 464$]$ is one of the classical theta-functions.

In his notebook [2, volume I, page 248], Ramanujan recorded several explicit values of theta-functions $\phi(q)$ and its quotients which are proved by Berndt and Chan [3]. They also found some new explicit values. An account of these can also be found in Berndt's book [4]. Yi [5] also evaluated many new values of $\phi(q)$ by finding explicit values of the parameters $h_{k, n}$ and $h_{k, n}^{\prime}$ for positive real numbers $k$ and $n$ which are defined by

$$
\begin{gathered}
h_{k, n}:=\frac{\phi(q)}{k^{1 / 4} \phi\left(q^{k}\right)}, \quad q=e^{-\pi \sqrt{n / k}}, \\
h_{k, n}^{\prime}:=\frac{\phi(-q)}{k^{1 / 4} \phi\left(-q^{k}\right)}, \quad q=e^{-2 \pi \sqrt{n / k}} .
\end{gathered}
$$

Yi [5] established several properties of these parameters and found their explicit values by appealing to transformation formulas and theta-function identities for $\phi(q)$. Recently, Saikia [6] found many new explicit values of quotients of $\phi(q)$ by finding explicit values of the parameter $A_{n}$ which is a particular case of the parameter $h_{k, n}$ where $k=4$. Saikia [6] also established some new theta-function identities for $\phi(q)$.

In the sequel of the previous work, in this paper we find some new explicit values of the parameters $h_{4, n}$ and $h_{2, n}$ which are particular cases of the parameter $h_{k, n}$ by using some properties of $h_{k, n}$ established in [5] and two new theta-function identities for $\phi(q)$. In addition, we give some applications of these new values of $h_{k, n}$ for the explicit evaluations of Ramanujan's continued fractions $c(q)$ and $K(q)$ defined by

$$
\begin{aligned}
& c(q):=\frac{1}{1+1-q^{2}} \frac{2 q}{q^{2}\left(1+q^{2}\right)^{2}} \frac{q^{4}\left(1+q^{4}\right)^{2}}{1-q^{6}}+, \\
& |q|<1, \\
& K(q):=\frac{q^{1 / 2}}{1+q_{+}} \frac{q^{2}}{1+q^{3}} \frac{q^{4}}{1+q^{5}}, \ldots \\
& |q|<1 \text {. }
\end{aligned}
$$

The continued fraction $c(q)$ is studied by Adiga and Anitha [7]. For explicit evaluations of $c(q)$, see [6]. The continued fraction $K(q)$ is called Ramanujan-Göllnitz-Gordon continued fraction [4, page 50]. For further references on $K(q)$, see $[8,9]$.

In Section 2, we record some preliminary results. Section 3 is devoted to prove two new identities for thetafunction $\phi(q)$. In Section 4, we find some new explicit values 
of the parameter $h_{4, n}$. In Section 5, we evaluate some new values of the parameter $h_{2, n}$. Finally in Section 6, we give applications of these values of $h_{4, n}$ and $h_{2, n}$ for the explicit evaluations of Ramanujan's continued fractions $c(q)$ and $K(q)$.

We end the introduction by defining Ramanujan's modular equation. The complete elliptic integral of the first kind $K(k)$ is defined by

$$
\begin{aligned}
K(k) & :=\int_{0}^{\pi / 2} \frac{d \phi}{\sqrt{1-k^{2} \sin ^{2} \phi}}=\frac{\pi}{2} \sum_{n=0}^{\infty} \frac{(1 / 2)_{n}^{2}}{(n !)^{2}} k^{2 n} \\
& =\frac{\pi}{2}{ }_{2} F_{1}\left(\frac{1}{2}, \frac{1}{2} ; 1 ; k^{2}\right),
\end{aligned}
$$

where $0<k<1,{ }_{2} F_{1}$ denotes the ordinary or Gaussian hypergeometric function, and

$$
(a)_{n}=a(a+1)(a+2) \cdots(a+n-1) .
$$

The number $k$ is called the modulus of $K$, and $k^{\prime}:=$ $\sqrt{1-k^{2}}$ is called the complementary modulus. Let $K, K^{\prime}, L$, and $L^{\prime}$ denote the complete elliptic integrals of the first kind associated with the moduli $k, k^{\prime}, l$, and $l^{\prime}$, respectively. Suppose that the equality

$$
n \frac{K^{\prime}}{K}=\frac{L^{\prime}}{L}
$$

holds for some positive integer $n$. Then a modular equation of degree $n$ is a relation between the moduli $k$ and $l$ which is implied by (7).

If we set

$$
q=\exp \left(-\pi \frac{K^{\prime}}{K}\right), \quad q^{\prime}=\exp \left(-\pi \frac{L^{\prime}}{L}\right),
$$

we see that (7) is equivalent to the relation $q^{n}=q^{\prime}$. Thus, a modular equation can be viewed as an identity involving theta-functions at the arguments $q$ and $q^{n}$. Ramanujan recorded his modular equations in terms of $\alpha$ and $\beta$, where $\alpha=k^{2}$ and $\beta=l^{2}$. We say that $\beta$ has degree $n$ over $\alpha$. The multiplier $m$ connecting $\alpha$ and $\beta$ is defined by $m=z_{1} / z_{n}$ where $z_{r}=\phi^{2}\left(q^{r}\right)$.

\section{Preliminary Results}

Lemma 1 (see [5, page 385, Theorem 2.2]). For positive real numbers $k$ and $n$,
(i) $h_{k, 1}=1$,
(ii) $h_{k, 1 / n}=1 / h_{k, n}$.

Lemma 2 (see [5, page 387, Corollary 2.6]). For positive real numbers $k$ and $n$,

$$
h_{k^{2}, n}=h_{k, n k} h_{k, n / k} \text {. }
$$

Lemma 3 (see [5, page 392, Theorem 4.6]). One has

$$
\sqrt{2}\left(h_{2,2 n} h_{2,4 n}+\frac{1}{h_{2,2 n} h_{2,4 n}}\right)=\frac{h_{2,4 n}}{h_{2, n}}+2,
$$

for any positive real number $n$.

Lemma 4 (see [10, page 122, Entry 10 (i) and (v)]). One has

(i) $\phi(q)=\sqrt{z}$,

(ii) $\left.\phi\left(q^{4}\right)=\sqrt{z}\left(1+(1-\alpha)^{1 / 4}\right)\right) / 2$.

Lemma 5 (see [10, page 280-281, Entry 13(vii)]). If $\beta$ has degree 5 over $\alpha$, then

$$
\begin{aligned}
& \left(\alpha \beta^{3}\right)^{1 / 8}+\left\{(1-\alpha)(1-\beta)^{3}\right\}^{1 / 8} \\
& \quad=\left(\alpha^{3} \beta\right)^{1 / 8}+\left\{(1-\alpha)^{3}(1-\beta)\right\}^{1 / 8}
\end{aligned}
$$

Lemma 6 (see [10, page 314, Entry 19(i)]). If $\beta$ has degree 7 over $\alpha$, then

$$
(\alpha \beta)^{1 / 8}+\{(1-\alpha)(1-\beta)\}^{1 / 8}=1 .
$$

\section{Two New Identities for $\phi(q)$}

In this section, we prove two new identities for thetafunction $\phi(q)$ by using Ramanujan's modular equations and transformation formulas.

Theorem 7. If $P=\phi(q) / \phi\left(q^{4}\right), Q=\phi\left(q^{5}\right) / \phi\left(q^{20}\right)$, then

$$
\begin{aligned}
& P^{6}-256 P Q+640 P^{2} Q-640 P^{3} Q \\
& +320 P^{4} Q-70 P^{5} Q+640 P Q^{2}-1600 P^{2} Q^{2} \\
& +1600 P^{3} Q^{2}-785 P^{4} Q^{2}+160 P^{5} Q^{2}-640 P Q^{3} \\
& +1600 P^{2} Q^{3}-1620 P^{3} Q^{3}+800 P^{4} Q^{3}-160 P^{5} Q^{3} \\
& +320 P Q^{4}-785 P^{2} Q^{4}+800 P^{3} Q^{4}-400 P^{4} Q^{4} \\
& +80 P^{5} Q^{4}-70 P Q^{5}+160 P^{2} Q^{5}-160 P^{3} Q^{5}+80 P^{4} Q^{5} \\
& -16 P^{5} Q^{5}+Q^{6}=0 .
\end{aligned}
$$

Proof. Transcribing $P$ and $Q$ using Lemma 4(i) and (iv) and then simplifying, we get

$$
(1-\alpha)^{1 / 4}=\frac{2}{P}-1, \quad(1-\beta)^{1 / 4}=\left(\frac{2}{Q}-1\right),
$$

where $\beta$ has degree 5 over $\alpha$.

Equivalently,

$$
\alpha=1-\left(\frac{2}{P}-1\right)^{4}, \quad \beta=1-\left(\frac{2}{Q}-1\right)^{4} .
$$


Now by Lemma 5, we have

$$
\begin{aligned}
& \left(\alpha \beta^{3}\right)^{1 / 8}-\left(\alpha^{3} \beta\right)^{1 / 8} \\
& \quad=\left\{(1-\alpha)^{3}(1-\beta)\right\}^{1 / 8}-\left\{(1-\alpha)(1-\beta)^{3}\right\}^{1 / 8} .
\end{aligned}
$$

Squaring (16) and simplifying, we arrive at

$$
\left(\alpha \beta^{3}\right)^{1 / 4}+\left(\alpha^{3} \beta\right)^{1 / 4}=x+2(\alpha \beta)^{1 / 2} \text {, }
$$

where

$$
\begin{aligned}
x= & \left\{(1-\alpha)^{3}(1-\beta)\right\}^{1 / 4}+\left\{(1-\alpha)(1-\beta)^{3}\right\}^{1 / 4} \\
& -2\{(1-\alpha)(1-\beta)\}^{1 / 2} .
\end{aligned}
$$

Squaring (17) and simplifying, we obtain

$$
\left(\alpha \beta^{3}\right)^{1 / 2}+\left(\alpha^{3} \beta\right)^{1 / 2}=y+4 x(\alpha \beta)^{1 / 2},
$$

where $y=x^{2}+2 \alpha \beta$.

Squaring (19) and simplifying, we obtain

$$
\alpha \beta^{3}+\alpha^{3} \beta+2 \alpha^{2} \beta^{2}-y^{2}-16 x^{2} \alpha \beta=8 x y(\alpha \beta)^{1 / 2} .
$$

Again squaring (20), we obtain

$$
\left(\alpha \beta^{3}+\alpha^{3} \beta+2 \alpha^{2} \beta^{2}-y^{2}-16 x^{2} \alpha \beta\right)^{2}-64 x^{2} y^{2} \alpha \beta=0 .
$$

Now employing (14) and (15) and factorizing using Mathematica, we deduce that

$$
f(P, Q) g(P, Q) h(P, Q)=0,
$$

where

$$
\begin{aligned}
f(P, Q)= & (P-Q)^{4} \\
g(P, Q)= & P^{6}-256 P Q+640 P^{2} Q \\
& -640 P^{3} Q+320 P^{4} Q-70 P^{5} Q+640 P Q^{2} \\
& -1600 P^{2} Q^{2}+1600 P^{3} Q^{2}-785 P^{4} Q^{2}+160 P^{5} Q^{2} \\
& -640 P Q^{3}+1600 P^{2} Q^{3}-1620 P^{3} Q^{3}+800 P^{4} Q^{3} \\
& -160 P^{5} Q^{3}+320 P Q^{4}-785 P^{2} Q^{4}+800 P^{3} Q^{4} \\
& -400 P^{4} Q^{4}+80 P^{5} Q^{4}-70 P Q^{5}+160 P^{2} Q^{5} \\
& -160 P^{3} Q^{5}+80 P^{4} Q^{5}-16 P^{5} Q^{5}+Q^{6}, \\
h(P, Q) \quad & \\
=16 P^{10} & -96 P^{9} Q-32 P^{10} Q+240 P^{8} Q^{2}+192 P^{9} Q^{2} \\
& +24 P^{10} Q^{2}-320 P^{7} Q^{3}-480 P^{8} Q^{3}-144 P^{9} Q^{3}
\end{aligned}
$$

$$
\begin{aligned}
& -8 P^{10} Q^{3}+256 P^{6} Q^{4}+608 P^{7} Q^{4}+384 P^{8} Q^{4} \\
& +40 P^{9} Q^{4}+P^{10} Q^{4}-448 P^{5} Q^{5}+352 P^{6} Q^{5} \\
& -1264 P^{7} Q^{5}+248 P^{8} Q^{5}-70 P^{9} Q^{5}+256 P^{4} Q^{6} \\
& +352 P^{5} Q^{6}-880 P^{6} Q^{6}+1640 P^{7} Q^{6}-785 P^{8} Q^{6} \\
& +160 P^{9} Q^{6}-320 P^{3} Q^{7}+608 P^{4} Q^{7}-1264 P^{5} Q^{7} \\
& +1640 P^{6} Q^{7}-1620 P^{7} Q^{7}+800 P^{8} Q^{7}-160 P^{9} Q^{7} \\
& +240 P^{2} Q^{8}-480 P^{3} Q^{8}+384 P^{4} Q^{8}+248 P^{5} Q^{8} \\
& -785 P^{6} Q^{8}+800 P^{7} Q^{8}-400 P^{8} Q^{8}+80 P^{9} Q^{8} \\
& -96 P Q^{9}+192 P^{2} Q^{9}-144 P^{3} Q^{9}+40 P^{4} Q^{9} \\
& -70 P^{5} Q^{9}+160 P^{6} Q^{9}-160 P^{7} Q^{9}+80 P^{8} Q^{9} \\
& -16 P^{9} Q^{9}+16 Q^{10}-32 P Q^{10}+24 P^{2} Q^{10} \\
& -8 P^{3} Q^{10}+P^{4} Q^{10}
\end{aligned}
$$

By examining the behavior of the first factor $f(P, Q)$ and the last factor $h(P, Q)$ of the left-hand side of $(22)$ near $q=$ 0 , it can be seen that there is a neighborhood about the origin, where these factors are not zero. Then the second factor $g(P, Q)$ is zero in this neighborhood. By the identity theorem, this factor is identically zero. Hence, we complete the proof.

Theorem 8. If $P=\phi(q) / \phi\left(q^{4}\right), Q=\phi\left(q^{7}\right) / \phi\left(q^{28}\right)$, then

$$
\begin{aligned}
& P^{8}-4096 P Q+14336 P^{2} Q-21504 P^{3} Q+17920 P^{4} Q \\
& -8736 P^{5} Q+2352 P^{6} Q-280 P^{7} Q+14336 P Q^{2} \\
& -51968 P^{2} Q^{2}+80640 P^{3} Q^{2}-69440 P^{4} Q^{2}+35056 P^{5} Q^{2} \\
& -9772 P^{6} Q^{2}+1176 P^{7} Q^{2}-21504 P Q^{3}+80640 P^{2} Q^{3} \\
& -129472 P^{3} Q^{3}+115360 P^{4} Q^{3}-60424 P^{5} Q^{3}+17528 P^{6} Q^{3} \\
& -2184 P^{7} Q^{3}+17920 P Q^{4}-69440 P^{2} Q^{4}+115360 P^{3} Q^{4} \\
& -106330 P^{4} Q^{4}+57680 P^{5} Q^{4}-17360 P^{6} Q^{4}+2240 P^{7} Q^{4} \\
& -8736 P Q^{5}+35056 P^{2} Q^{5}-60424 P^{3} Q^{5}+57680 P^{4} Q^{5} \\
& -32368 P^{5} Q^{5}+10080 P^{6} Q^{5}-1344 P^{7} Q^{5}+2352 P Q^{6} \\
& -9772 P^{2} Q^{6}+17528 P^{3} Q^{6}-17360 P^{4} Q^{6}+10080 P^{5} Q^{6} \\
& -3248 P^{6} Q^{6}+448 P^{7} Q^{6}-280 P Q^{7}+1176 P^{2} Q^{7} \\
& -2184 P^{3} Q^{7}+2240 P^{4} Q^{7}-1344 P^{5} Q^{7}+448 P^{6} Q^{7} \\
& -64 P^{7} Q^{7}+Q^{8}=0
\end{aligned}
$$


Proof. Transcribing $P$ and $Q$ using Lemma 4(i) and (iv) and then simplifying, we obtain

$$
(1-\alpha)^{1 / 4}=\frac{2}{P}-1, \quad(1-\beta)^{1 / 4}=\left(\frac{2}{Q}-1\right),
$$

where $\beta$ has degree 7 over $\alpha$.

Equivalently,

$$
\alpha=1-\left(\frac{2}{P}-1\right)^{4}, \quad \beta=1-\left(\frac{2}{Q}-1\right)^{4} .
$$

Now by Lemma 6, we have

$$
\{(1-\alpha)(1-\beta)\}^{1 / 8}=1-(\alpha \beta)^{1 / 8} .
$$

Squaring (27) and simplifying, we obtain

$$
x-(\alpha \beta)^{1 / 4}=-2(\alpha \beta)^{1 / 8},
$$

where $x=\{(1-\alpha)(1-\beta)\}^{1 / 4}-1$.

Squaring (28) and simplifying, we obtain

$$
x^{2}+(\alpha \beta)^{1 / 2}=(4+2 x)(\alpha \beta)^{1 / 4} \text {. }
$$

Squaring (29) and simplifying, we obtain

$$
x^{4}+\alpha \beta=\left((4+2 x)^{2}-2 x^{2}\right)(\alpha \beta)^{1 / 2} \text {. }
$$

Again, squaring (30), we arrive at

$$
\left(x^{4}+\alpha \beta\right)^{2}=\left((4+2 x)^{2}-2 x^{2}\right)^{2}(\alpha \beta) \text {. }
$$

Employing (25) and (26) in (31) and simplifying with the help of Mathematica, we complete the proof.

\section{New Values of $h_{4, n}$}

In this section, we find some new values of $h_{4, n}$ and $h_{2, n}$ by using theta-function identities proved in Section 3 and the properties of $h_{k, n}$ listed in Lemmas 1 and 2 . We begin with following remarks.

Remark 9. The values of $h_{k, n}$ are real and $h_{k, n}<1$ for all $n>1$ if $k>1$. We also note that the values of $h_{k, n}$ decrease as $n$ increases when $k>1$. In view of this, in the following theorem we have $h_{4,4}>h_{4,5}>h_{4,7}>h_{4,8}>h_{4,25}>h_{4,49}$ where $h_{4,4}$ and $h_{4,8}$ are evaluated in [6, Theorem 4.3]. Yi [5] also evaluated the value of $h_{4,4}$.

\section{Theorem 10. One has}

$$
\begin{aligned}
& \text { (i) } h_{4,5}=(\sqrt{22+10 \sqrt{5}}-\sqrt{2}-\sqrt{10}- \\
& \sqrt{\left(\sqrt{2}+\sqrt{10}-{\left.\sqrt{22+10 \sqrt{5}})^{2}-4\right)}^{2},\right.} \\
& \text { (ii) } h_{4,1 / 5}=(\sqrt{22+10 \sqrt{5}}-\sqrt{2}-\sqrt{10}+ \\
& \sqrt{\left(\sqrt{2}+\sqrt{10}-\sqrt{22+10 \sqrt{5}}^{2}-4\right) / 2}
\end{aligned}
$$

(iii) $h_{4,25}=114-80 \sqrt{2}+\sqrt{25965-18360 \sqrt{2}}-$

$$
\sqrt{\left(114-80 \sqrt{2}+\sqrt{25965-18360 \sqrt{2}}^{2}-1\right.}
$$

(iv) $h_{4,1 / 25}=114-80 \sqrt{2}+\sqrt{25965-18360 \sqrt{2}}+$

$$
\sqrt{\left(114-80 \sqrt{2}+\sqrt{25965-18360 \sqrt{2}}^{2}-1\right.} \text {, }
$$

(v) $h_{4,7}=(12-7 \sqrt{2}-\sqrt{7(34-24 \sqrt{2})}) / 2$,

(vi) $h_{4,1 / 7}=(12-7 \sqrt{2}+\sqrt{7(34-24 \sqrt{2})}) / 2$,

(vii) $h_{4,49}=a+\sqrt{b}-\sqrt{-1+(a+\sqrt{b})^{2}}$,

(viii) $h_{4,1 / 49}=a+\sqrt{b}+\sqrt{-1+(a+\sqrt{b})^{2}}$,

where $a=1317-931 \sqrt{2}+\sqrt{3470684-2454144 \sqrt{2}}$ and $b=6940535-4907700 \sqrt{2}+$ $1306133196 \sqrt{7 /(123953-87648 \sqrt{2})}-$ $923575640 \sqrt{14 /(123953-87648 \sqrt{2})}$.

Proof. For (i) and (ii), setting $k=4$ and employing the definition of $h_{k, n}$ in Theorem 7 , we get

$$
P=\sqrt{2} h_{4, n}, \quad Q=\sqrt{2} h_{4,25 n} .
$$

Setting $n=1 / 5$ in (32), applying to (13), and then simplifying using Lemma 1(ii), we obtain

$$
\begin{aligned}
& \left(h_{4,5}^{6}+h_{4,5}^{-6}\right)-70\left(h_{4,5}^{4}+h_{4,5}^{-4}\right)+320 \sqrt{2}\left(h_{4,5}^{3}+h_{4,5}^{-3}\right) \\
& \quad-1425\left(h_{4,5}^{2}+h_{4,5}^{-2}\right)+1920 \sqrt{2}\left(h_{4,5}+h_{4,5}^{-1}\right)-3348=0 .
\end{aligned}
$$

Equivalently,

$$
A^{6}-76 A^{4}+320 \sqrt{2} A^{3}-1136 A^{2}+960 \sqrt{2} A-640=0,
$$

where

$$
A:=h_{4,5}+h_{4,5}^{-1}
$$

Solving (34) for $A$ and noting that $A$ has positive real value greater than 1 , we obtain

$$
A=-\sqrt{2}-\sqrt{10}+\sqrt{22+10 \sqrt{5}}
$$

Invoking (36) in (35), solving for $h_{4,5}$, and using the fact in Remark 9, we complete the proof of (i). Noting $h_{4,1 / 5}=1 / h_{4,5}$ from Lemma 1(ii), we arrive at (ii).

For proofs of (iii) and (iv), we set $n=1$ in (32), applying to (13) and then simplifying using Lemma 1(i), we arrive at

$$
\begin{aligned}
& \left(h_{4,25}^{2}+h_{4,25}^{-2}\right)-(456-320 \sqrt{2})\left(h_{4,25}+h_{4,25}^{-1}\right) \\
& -(674-840 \sqrt{2})=0 .
\end{aligned}
$$

Equivalently,

$$
B^{2}+8(-57+40 \sqrt{2}) B+(-674+840 \sqrt{2})=0,
$$


where

$$
B:=h_{4,25}+h_{4,25}^{-1} \text {. }
$$

Solving (38) for $B$ and noting that $B$ has positive real value greater than 1 , we obtain

$$
B=2(114-80 \sqrt{2}+\sqrt{5(5193-3672 \sqrt{2})}) .
$$

Invoking (40) in (39), solving for $h_{4,25}$, and using the fact in Remark 9, we complete the proof of (iii). Noting $h_{4,1 / 25}=$ $1 / h_{4,25}$ from Lemma 1 (iv), we prove (ii).

To prove (v) and (vi), applying the definition of $h_{4, n}$ in Theorem 8, we get

$$
P=\sqrt{2} h_{4, n}, \quad Q=\sqrt{2} h_{4,49 n} .
$$

Setting $n=1 / 7$ in (41), applying in (24), and then simplifying using Lemma 1(ii), we arrive at

$$
\begin{aligned}
h_{4,7}^{16} & -280 h_{4,7}^{14}+2352 \sqrt{2} h_{4,7}^{13}-18508 h_{4,7}^{12} \\
& +44016 \sqrt{2} h_{4,7}^{11}-140616 h_{4,7}^{10}+159264 \sqrt{2} h_{4,7}^{9} \\
& -262810 h_{4,7}^{8}+159264 \sqrt{2} h_{4,7}^{7}-140616 h_{4,7}^{6} \\
& +44016 \sqrt{2} h_{4,7}^{5}-18508 h_{4,7}^{4}+2352 \sqrt{2} h_{4,7}^{3} \\
& -280 h_{4,7}^{2}+1=0 .
\end{aligned}
$$

Dividing (42) by $h_{4,7}^{8}$ and simplifying, we get

$$
\begin{aligned}
& \left(h_{4,7}^{8}+h_{4,7}^{-8}\right)-280\left(h_{4,7}^{6}+h_{4,7}^{-6}\right)+2352 \sqrt{2}\left(h_{4,7}^{5}+h_{4,7}^{-5}\right) \\
& -18508\left(h_{4,7}^{4}+h_{4,7}^{-4}\right)+44016 \sqrt{2}\left(h_{4,7}^{3}+h_{4,7}^{-3}\right) \\
& -140616\left(h_{4,7}^{2}+h_{4,7}^{-2}\right)+159264 \sqrt{2}\left(h_{4,7}+h_{4,7}^{-1}\right) \\
& -262810=0 .
\end{aligned}
$$

Equivalently,

$$
\begin{aligned}
& L^{8}-288 L^{6}+2352 \sqrt{2} L^{5}-16808 L^{4}+32256 \sqrt{2} L^{3}-69120 L^{2} \\
& \quad+38976 \sqrt{2} L-18032=0,
\end{aligned}
$$

where

$$
L:=h_{4,7}+h_{4,7}^{-1}
$$

Solving (44) by using Mathematica and noting that $L$ has positive real value greater that 1 satisfying the fact in Remark 9, we obtain

$$
L=12-7 \sqrt{2} \text {. }
$$

Employing (46) in (45), solving for $h_{4,7}$, and using the fact in Remark 9, we complete the proof of $(\mathrm{v})$. Noting $h_{4,1 / 7}=$ $1 / h_{4,7}$, we arrive at (vi).
For proofs of (vii) and (viii), setting $n=1$ in (41), applying in (24), and simplifying using Lemma 1(ii), we arrive at

$$
\begin{aligned}
& h_{4,49}^{4}+h_{4,49}^{-4}+4(-1317+931 \sqrt{2})\left(h_{4,49}^{3}+h_{4,49}^{-3}\right) \\
& \quad+28(-2053+1452 \sqrt{2})\left(h_{4,49}^{2}+h_{4,49}^{-2}\right) \\
& \quad+56(-2409+1703 \sqrt{2})\left(h_{4,49}+h_{4,49}^{-1}\right) \\
& \quad+210(-837+592 \sqrt{2})=0 .
\end{aligned}
$$

Equivalently,

$$
\begin{aligned}
& D^{4}+8(-1317+931 \sqrt{2}) D^{3}+16(-3593+2541 \sqrt{2}) D^{2} \\
& +64(-1614+1141 \sqrt{2}) D+128(-475+336 \sqrt{2})=0
\end{aligned}
$$

where

$$
D=h_{4,49}+h_{4,49}^{-1} \text {. }
$$

Solving (48) for $D$ and noting that $D$ has positive real value greater than 1 , we obtain

$$
D=2(a+\sqrt{b})
$$

where $a=1317-931 \sqrt{2}+\sqrt{3470684-2454144 \sqrt{2}}$ and $b=6940535-4907700 \sqrt{2}+$ $1306133196 \sqrt{7 /(123953-87648 \sqrt{2})}-$ $923575640 \sqrt{14 /(123953-87648 \sqrt{2})}$.

Invoking (50) in (49), solving for $h_{4,49}$, and using the fact in Remark 9, we arrive at (vi). Noting $h_{4,1 / 49}=1 / h_{4,49}$ from Lemma 1(ii), we complete the proof of (vii).

\section{New Values of $h_{2, n}$}

In this section, we find some new values of the parameter $h_{2, n}$ by using the values of $h_{4, n}$ evaluated in Section 4 and in [6].

Theorem 11. One has

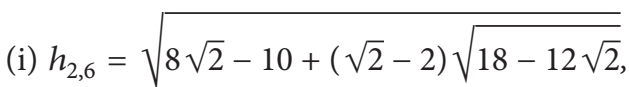

(ii) $h_{2,3 / 2} \quad=\quad\left(2 \quad-\quad \begin{array}{lll}2 & -\end{array}\right.$ $\sqrt{18-12 \sqrt{2}}) /(2 \sqrt{8 \sqrt{2}-10+(\sqrt{2}-2) \sqrt{18-12 \sqrt{2}})}$,

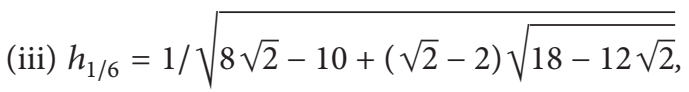

(iv) $h_{2,2 / 3}=2 \sqrt{8 \sqrt{2}-10+(\sqrt{2}-2) \sqrt{18-12 \sqrt{2}}} /(2-$ $\sqrt{2}+\sqrt{18-12 \sqrt{2}})$.

Proof. Setting $k=2$ and $n=3$ in Lemma 2, we deduce that

$$
h_{4,3}=h_{2,6} h_{2,3 / 2} \text {. }
$$


From [6, page 174, Theorem 4.3(vii)], we have

$$
h_{4,3}=\frac{(2-\sqrt{2}+\sqrt{18-12 \sqrt{2}})}{2} .
$$

Combining (51) and (52), we obtain

$$
h_{2,6} h_{2,3 / 2}=\frac{(2-\sqrt{2}+\sqrt{18-12 \sqrt{2}})}{2} .
$$

Next, setting $n=1 / 6$ in Lemma 3 and simplifying using Lemma 1(ii), we obtain

$$
\sqrt{2}\left(h_{2,6} h_{2,3 / 2}+\left(h_{2,6} h_{2,3 / 2}\right)^{-1}\right)=\left(\frac{h_{2,6}}{h_{2,3 / 2}}\right)+2 \text {. }
$$

Invoking (53) in (54) and simplifying, we deduce that

$$
\left(\frac{h_{2,6}}{h_{2,3 / 2}}\right)=\frac{2(8 \sqrt{2}-10+(\sqrt{2}-2) \sqrt{18-12 \sqrt{2}})}{2-\sqrt{2}+\sqrt{18-12 \sqrt{2}}} .
$$

Multiplying (53) and (55) and simplifying, we complete the proof of (i). Dividing (53) by (55) and simplifying, we arrive at (ii). (iii) and (iv) follow from (i) and (ii), respectively, and Lemma 1(ii).

The proofs of Theorems $12-15$ are identical to the proof of Theorem 11. So we omit details and give only references of the required results to prove them.

Theorem 12. One has

(i) $h_{2,10}=\sqrt{\left(4+c^{2}\right) \sqrt{2}-4 c / 2}$,

(ii) $h_{2,5 / 2}=c / \sqrt{\left(4+c^{2}\right) \sqrt{2}-4 c}$,

(iii) $h_{2,1 / 10}=2 / \sqrt{\left(4+c^{2}\right) \sqrt{2}-4 c}$,

(iv) $h_{2,2 / 5}=\sqrt{\left(4+c^{2}\right) \sqrt{2}-4 c / c}$,

where $c=\sqrt{22+10 \sqrt{5}}-\sqrt{2}-\sqrt{10}-$

Proof of Theorem 12 follows from Theorem 10(i), Lemma 2 with $k=2$ and $n=5$, Lemma 1(ii), and Lemma 3 with $n=1 / 10$.

Theorem 13. One has

(i) $h_{2,50}=\sqrt{-2 d+\sqrt{2}\left(d^{2}+1\right)}$,

(ii) $h_{2,25 / 2}=d / \sqrt{-2 d+\sqrt{2}\left(d^{2}+1\right)}$,

(iii) $h_{2,1 / 50}=1 / \sqrt{-2 d+\sqrt{2}\left(d^{2}+1\right)}$, (iv) $h_{2,2 / 25}=\sqrt{-2 d+\sqrt{2}\left(d^{2}+1\right)} / d$,

where $d=114-80 \sqrt{2}+\sqrt{25965-18360 \sqrt{2}}-$

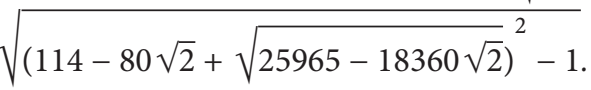

To prove Theorem 13, we use Theorem 10(iii), Lemma 2 with $k=2$ and $n=25$, Lemma 1(ii), and Lemma 3 with $n=$ $1 / 50$.

Theorem 14. One has

(i) $h_{2,14}=\sqrt{(-8+6 \sqrt{2})(12-7 \sqrt{2}-\sqrt{238-168 \sqrt{2}})}$,

(ii) $h_{2,7 / 2}=\sqrt{12-7 \sqrt{2}-\sqrt{238-168 \sqrt{2}}} / 2 \sqrt{-8+6 \sqrt{2}}$,

(iii) $h_{2,1 / 14}=1 / \sqrt{(-8+6 \sqrt{2})(12-7 \sqrt{2}-\sqrt{238-168 \sqrt{2}})}$,

(iv) $h_{2,2 / 7}=2 \sqrt{-8+6 \sqrt{2}} / \sqrt{12-7 \sqrt{2}-\sqrt{238-168 \sqrt{2}}}$.

We employ Theorem 10(v), Lemma 2 with $k=2$ and $n=7$, Lemma 1 (ii), and Lemma 3 with $n=1 / 14$ to prove Theorem 14

Theorem 15. One has

(i) $h_{2,98}=\sqrt{2\left(a+\sqrt{b}-\sqrt{(a+\sqrt{b})^{2}-1}\right)(\sqrt{2 a}+\sqrt{2 b}-1)}$,

(ii) $h_{2,49 / 2}=\sqrt{\left(a+\sqrt{b}-\sqrt{(a+\sqrt{b})^{2}-1}\right) / 2(\sqrt{2 a}+\sqrt{2 b}-1)}$,

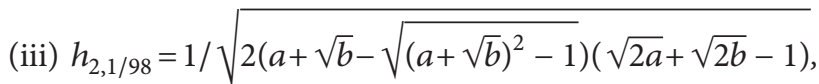

(iv) $h_{2,2 / 49}=\sqrt{2(\sqrt{2 a}+\sqrt{2 b}-1) /\left(a+\sqrt{b}-\sqrt{(a+\sqrt{b})^{2}-1}\right)}$, where $a$ and $b$ are given in Theorem 10(viii).

Proof follows from Theorem 10(vii), Lemma 2 with $k=2$ and $n=49$, Lemma 1(ii), and Lemma 3 with $n=1 / 98$.

\section{Applications of $h_{4, n}$ and $h_{2, n}$}

In this section, we use the new values of the parameters $h_{4, n}$ and $h_{2, n}$ to find explicit values of continued fractions $c(q)$ and $K(q)$ defined in (3) and (4), respectively.

The parameter $h_{4, n}$ is useful in finding explicit values of the continued fraction $c(q)$. If we know values of the parameter $h_{4, n}$ for any positive real number $n$, then explicit values of $c\left(e^{-\pi \sqrt{n} / 2}\right)$ can be calculated by appealing to the following theorem.

Theorem 16 (see [6, page 177, Theorem 5.1]). One has

$$
c\left(e^{-\pi \sqrt{n} / 2}\right)=\frac{1}{\sqrt{2} h_{4, n}},
$$

where $n$ is any positive real number. 
For example, employing the value of $h_{4,5}$ from Theorem 10(i) in Theorem 16, we obtain

$$
\begin{aligned}
& c\left(e^{-\pi \sqrt{5} / 2}\right) \\
&=\sqrt{2} \times(\sqrt{22+10 \sqrt{5}}-\sqrt{2}-\sqrt{10} \\
& \\
&-\sqrt{\left.(\sqrt{2}+\sqrt{10}-\sqrt{22+10 \sqrt{5}})^{2}-4\right)^{-1} .} .
\end{aligned}
$$

Similarly, we can find new values of $c\left(e^{-\pi / 2 \sqrt{5}}\right), c\left(e^{-5 \pi / 2}\right)$, $c\left(e^{-\pi / 10}\right), c\left(e^{-\pi \sqrt{7} / 2}\right), c\left(e^{-\pi / 2 \sqrt{7}}\right), c\left(e^{-7 \pi / 2}\right)$, and $c\left(e^{-\pi / 14}\right)$ by employing the values of $h_{4, n}$ from Theorem 10(ii)-(viii), respectively, in Theorem 16 . Since it is a routine calculation, we omit details.

Next, the parameter $h_{2, n}$ is connected to continued fraction $K(q)$ by the following theorem.

Theorem 17 (see [8, page 281, Theorem 4.1]). For any positive real number $n$, one has

$$
K^{2}\left(e^{-\pi \sqrt{n / 2}}\right)=\frac{2^{1 / 4} h_{2, n}-1}{2^{1 / 4} h_{2, n}+1} .
$$

From Theorem 17, we note that if the values of $h_{2, n}$ are known, then the values of $K\left(e^{-\pi \sqrt{n / 2}}\right)$ can easily be evaluated. For example, using the value of $h_{2,6}$ from Theorem 11(i) in Theorem 17, we evaluate

$$
\begin{aligned}
& K\left(e^{-\pi \sqrt{6 / 2}}\right) \\
& \quad=\sqrt{\frac{2^{1 / 4} \sqrt{8 \sqrt{2}-10+(\sqrt{2}-2) \sqrt{18-12 \sqrt{2}}}-1}{2^{1 / 4} \sqrt{8 \sqrt{2}-10+(\sqrt{2}-2) \sqrt{18-12 \sqrt{2}}}+1} .} .
\end{aligned}
$$

Similarly, we can evaluate new values of $K\left(e^{-\pi \sqrt{n / 2}}\right)$ for $n=3 / 2,1 / 6,2 / 3,10,5 / 2,1 / 10,2 / 5,50,25 / 2,1 / 50,2 / 25,14$, $7 / 2,1 / 14,2 / 7,98,49 / 2$, and $2 / 49$ by using Theorem 17 and the values of $h_{2, n}$ evaluated in Theorems 11-15.

\section{Acknowledgment}

The author is thankful to the University Grants Commission, New Delhi, India for partially supporting the research work under the Grant no. F. No. 41-1394/2012(SR).

\section{References}

[1] E. T. Whittaker and G. N. Watson, A Course of Modern Analysis, Cambridge Mathematical Library, Cambridge University Press, Cambridge, UK, 1996, Indian edition is published by Universal Book Stall, New Delhi, India, 1991.
[2] S. Ramanujan, Notebooks (2 Volumes), Tata Institute of Fundamental Research, Mumbai, India, 1957.

[3] B. C. Berndt and H. H. Chan, "Ramanujan's explicit values for the classical theta-function," Mathematika, vol. 42, no. 2, pp. 278-294, 1995.

[4] B. C. Berndt, Ramanujan's Notebooks, Part V, Springer, New York, NY, USA, 1998.

[5] J. Yi, "Theta-function identities and the explicit formulas for theta-function and their applications," Journal of Mathematical Analysis and Applications, vol. 292, no. 2, pp. 381-400, 2004.

[6] N. Saikia, "Some new identities for Ramanujan's theta-function $\phi(q)$ and applications," Far East Journal of Mathematical Sciences, vol. 44, no. 2, pp. 167-179, 2010.

[7] C. Adiga and N. Anitha, "A note on a continued fraction of Ramanujan," Bulletin of the Australian Mathematical Society, vol. 70, no. 3, pp. 489-497, 2004.

[8] N. D. Baruah and N. Saikia, "Explicit evaluations of RamanujanGöllnitz-Gordon continued fraction," Monatshefte für Mathematik, vol. 154, no. 4, pp. 271-288, 2008.

[9] H. H. Chan and S.-S. Huang, "On the Ramanujan-GöllnitzGordon continued fraction," The Ramanujan Journal, vol. 1, no. 1, pp. 75-90, 1997.

[10] B. C. Berndt, Ramanujan's Notebooks, Part III, Springer, New York, NY, USA, 1991. 


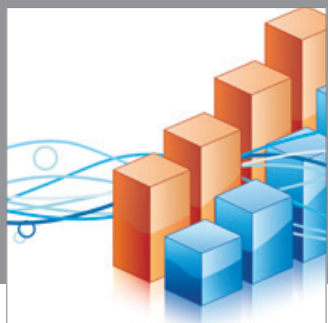

Advances in

Operations Research

mansans

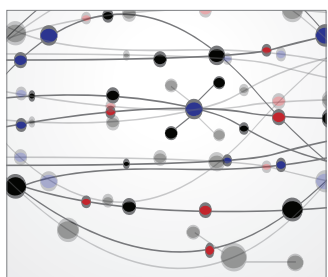

The Scientific World Journal
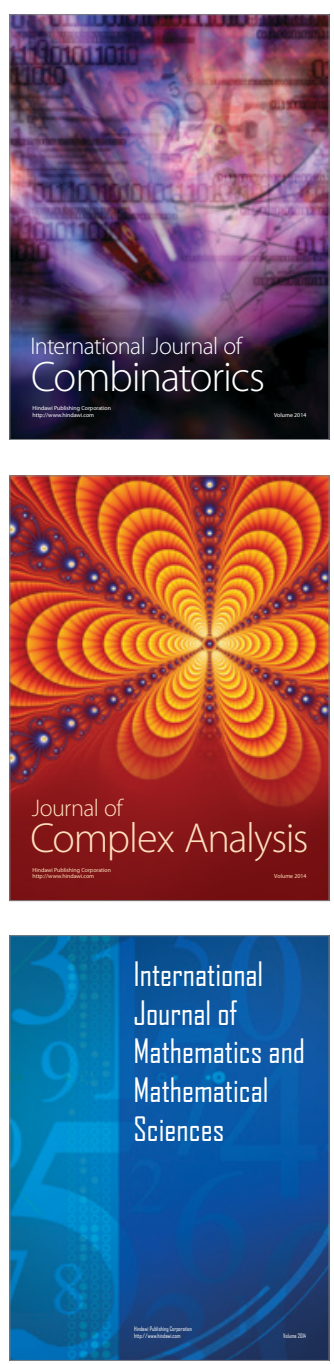
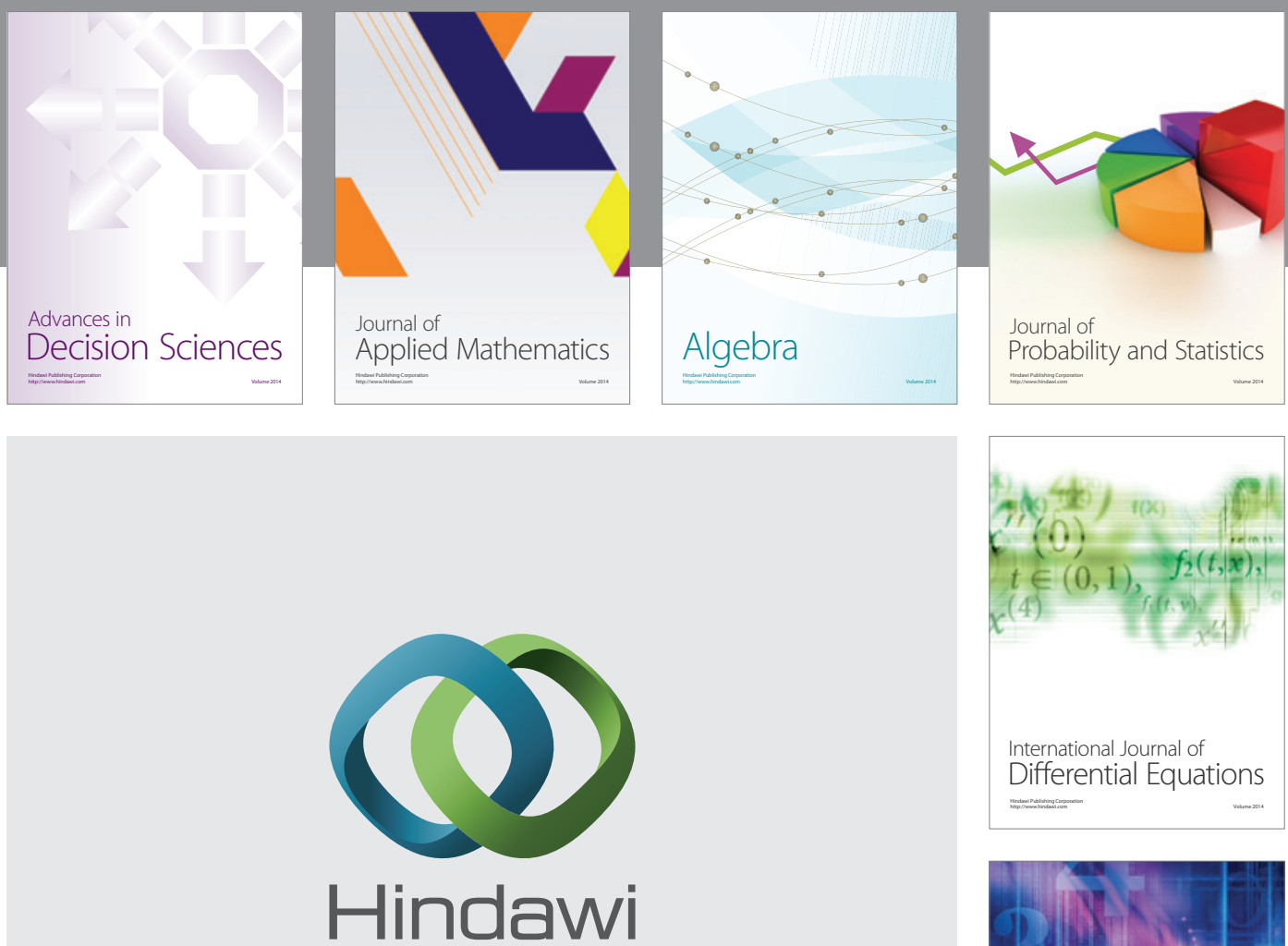

Submit your manuscripts at http://www.hindawi.com
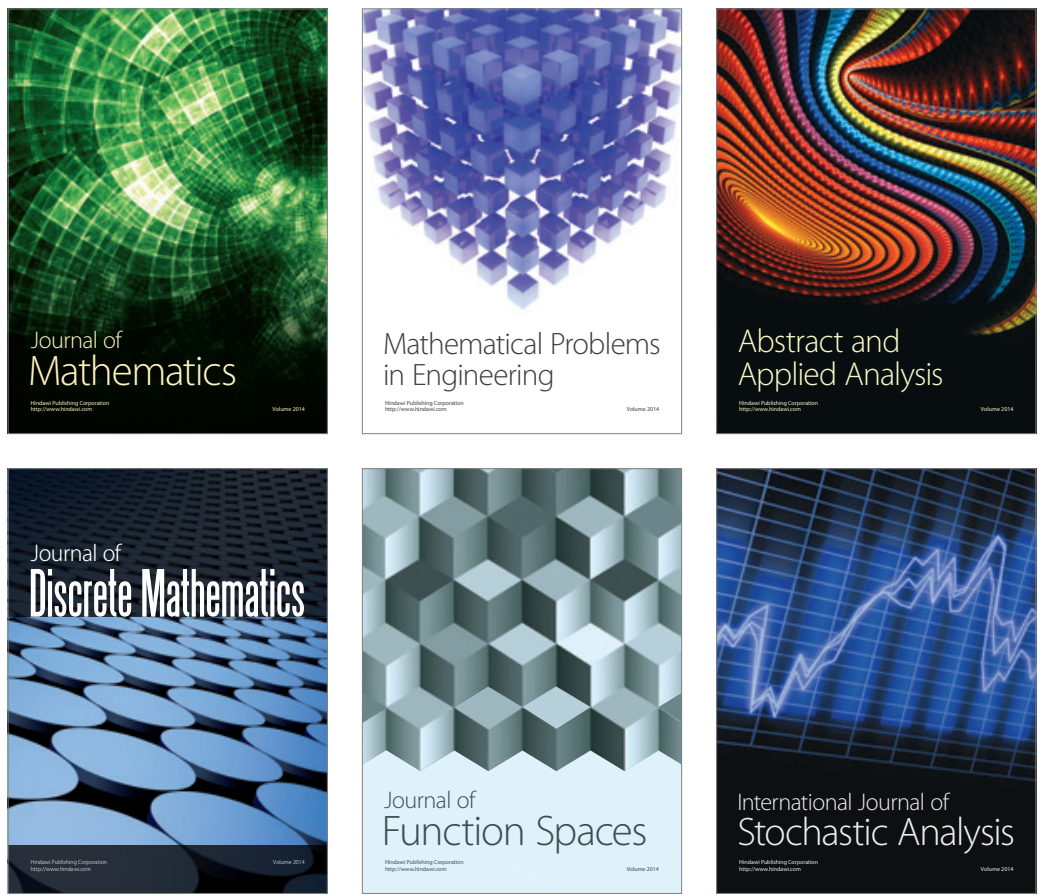

Journal of

Function Spaces

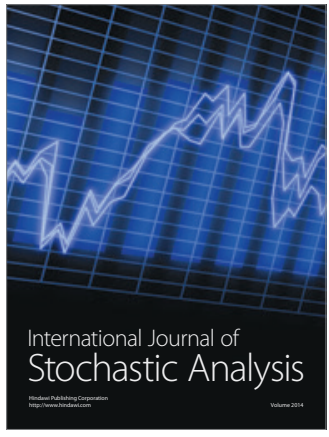

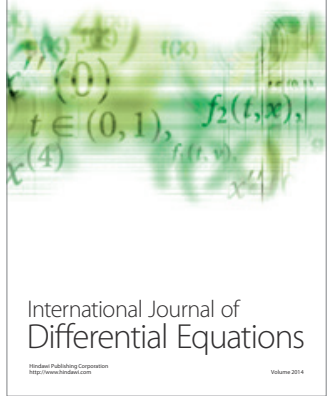
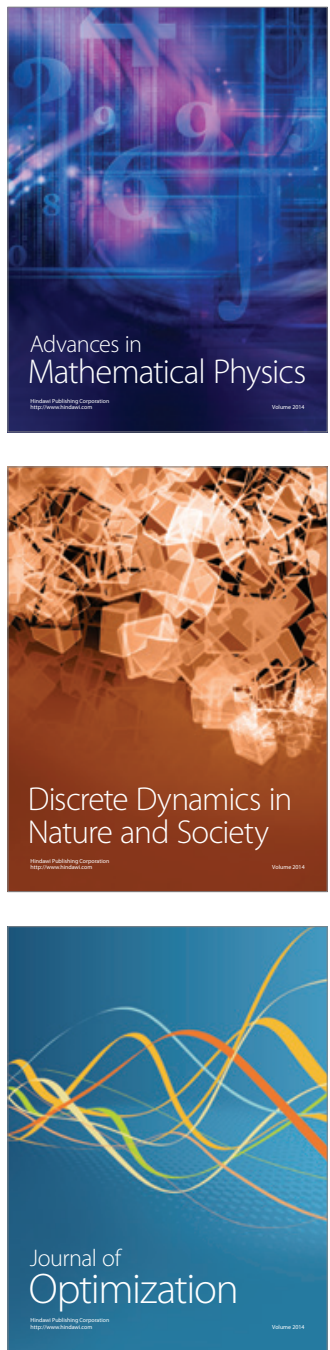